\title{
Interfaces with a single growth inhomogeneity and anchored boundaries
}

\author{
M. D. Grynberg \\ Departamento de Física, Universidad Nacional de La Plata, (1900) La Plata, Argentina
}

(Received 11 April 2003; published 3 October 2003)

\begin{abstract}
The dynamics of a one-dimensional growth model involving attachment and detachment of particles is studied in the presence of a localized growth inhomogeneity along with anchored boundary conditions. At large times, the latter enforce an equilibrium stationary regime which allows for an exact calculation of roughening exponents. The stochastic evolution is related to a spin Hamiltonian whose spectrum gap embodies the dynamic scaling exponent of late stages. For vanishing gaps the interface can exhibit a slow morphological transition followed by a change of scaling regimes which are studied numerically. Instead, a faceting dynamics arises for gapful situations.
\end{abstract}

DOI: 10.1103/PhysRevE.68.041603

\section{INTRODUCTION}

After two decades of investigations the dynamics of growing interfaces continues to be a subject of enormous interest, providing a framework to compare experiments, simulations, and theory, let alone the wide range of applications encountered [1]. Despite the vast diversity of morphologies in which growing surfaces can evolve, most numerical analysis and theoretical studies pointed out the onset of scaling regimes emerging at both large time and length scales. This enabled a classification of apparently dissimilar processes in terms of universality classes characterized by a set of scaling exponents which dominate the late evolution stages [1,2].

A common feature associated with these processes is the emergence of rather slow temporal crossovers in which the early dynamics exhibits quite different roughening characteristics from those observed in the asymptotic limit [3]. The presence of growth rate inhomogeneities or growth defects localized within small spatial regions of the substrate plane (columnar defects) is one of the simplest mechanisms whereby such crossovers can be observed $[1,4]$. Another possibility is realized by anchoring conditions through which nonequilibrium fluctuations are completely suppressed at the interface boundaries [5]. The main interest in those situations is in the morphological phase transitions that may occur at large times. In this work we investigate the change of scaling regimes accompanying these transitions by means of a prototype restricted solid on solid (RSOS) growth model [6,7] combining both of these mechanisms in one dimension. As we shall see, anchored boundaries are essential for the appearance of equilibrium regimes which in turn allow for a simple calculation of roughening exponents at late stages.

Morphological transitions in confined geometries actually occur in flexible manifolds characterizing physical phenomena as diverse as the unbinding of a directed polymer from a wall [8], and the depinning of a flux line from a dislocation in a type II superconductor [9]. At the phenomenological level of the Kardar-Parisi-Zhang equation [10], thoroughly studied in the continuum theory of kinetic roughening, large scale morphology changes can be accounted for by assuming a growth velocity that is a symmetric function of the local inclination of the interface [4], so the latter can increase its growth rate by adopting a nonvanishing tilt. Therefore, a
PACS number(s): 81.15.Aa, 05.40.-a, 02.50.-r, 75.10.Jm

macroscopic hill can emerge in response to a sufficiently large growth rate inhomogeneity as this morphology grows faster than the planar interface, in turn allowing the system to accommodate the external bias [1]. Here, we show that a similar scenario may also arise under equilibrium and near equilibrium conditions dominated by unusual scaling regimes.

In studying the latter it is useful to consider the mean square fluctuations of the average interface height $\bar{h}(t)$ which yields a measure of the interface width

$$
W^{2}(L, t)=\frac{1}{L} \sum_{n}\left\langle\left[h_{n}(t)-\bar{h}(t)\right]^{2}\right\rangle
$$

where the brackets denote an ensemble average over all possible evolutions of heights $\left\{h_{n}\right\}$ forming the interface at time $t$, which grows on a substrate of size $L$. On general grounds it can be argued that $W$ scales as [11]

$$
W(L, t)=L^{\zeta} f\left(t / L^{z}\right)
$$

where the scaling function $f(c)$ satisfies

$$
f(c) \sim\left\{\begin{array}{cc}
c^{\zeta / z} & \text { for } \quad c \ll 1, \\
\text { const } & \text { for } \quad c \gg 1 .
\end{array}\right.
$$

Hence, it follows that finite systems saturate as $W \propto L^{\zeta}$, whereas in the thermodynamic limit the asymptotic growth is ruled by the exponent $\beta=\zeta / z$, that is, $W \propto t^{\beta}$. The exponent $\zeta$ describes the roughness dependence of the interface width on the typical substrate size. In turn the exponent $z$, often known as the dynamic exponent, gives the fundamental scaling between length and time.

In practice, Eqs. (2) and (3) yield a standard procedure which is often followed to extract and corroborate scaling exponents predicted by other approaches and certainly we will make use of this hypothesis as well. However, due to the presence of the crossovers referred to above, a complementary procedure would be needed if the former becomes exceptionally slow. To this aim, we will also exploit the known equivalence between the RSOS growth models already mentioned and a one-dimensional gas of hard-core particles undergoing an asymmetric exclusion process $[1,6,7]$ (see Fig. 1 ). The idea is to cast the evolution operator of the associated 


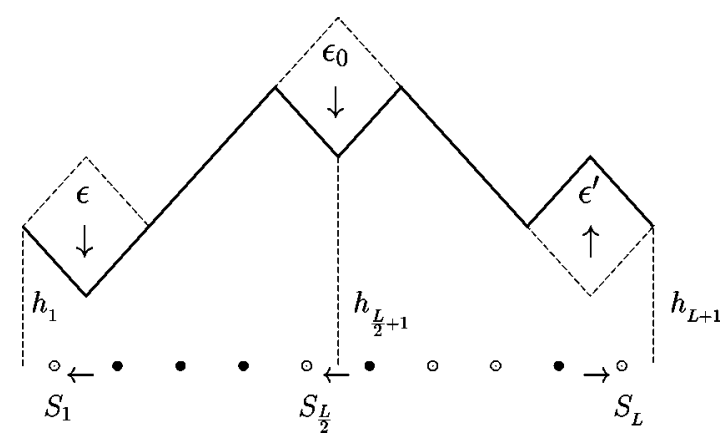

FIG. 1. Schematic representation of monomer depositionevaporation onto a RSOS interface with anchored boundaries at $h_{1}=h_{L+1}$. The equivalent spin- $\frac{1}{2}\left(s_{n} \equiv h_{n+1}-h_{n}\right)$ or hard-core particle dynamics involves a left (right) particle hopping with rate $\epsilon$ $\left(\epsilon^{\prime}\right)$ for monomer adsorption (desorption). The corresponding rates for the inhomogeneity at $h_{L / 2+1}$ are $\epsilon_{0}$ and $\epsilon_{0}^{\prime}$.

master equation of this latter process [12] into a suitable quantum spin representation $[13,14]$ lending itself more readily for a finite size scaling analysis. Since the dynamic exponent $z$ of Eq. (2) is ultimately embodied in the gap behavior of the evolution operator (or spin "Hamiltonian"), the study of its lower spectrum can then provide information of the late evolution stages in a more direct manner. Evidently, this methodology along with the evaluation of the roughening exponent $\zeta$ - simplified greatly by the anchoring boundary conditions - circumvents the problem of arbitrarily long transient regimes though on the other hand is limited severely by the affordable system sizes. A posteriori, it will turn out that already modest lengths can yield clear finite size trends. This strategy will be explained in Sec. II and its results compared with those of standard techniques given in Sec. III. We end the paper with Sec. IV which contains our conclusions, along with some remarks on extensions of this work.

\section{SPIN REPRESENTATION}

Let us consider the dynamics of lattice aggregation models with no overhangs, including both adsorption and desorption of monomers at random locations of a one-dimensional interface [6,7], such as that described in Fig. 1. As usual, on a coarse grained level of description the state of a surface at a given time is represented by a set of single-valued functions $h_{n}(t)$ measuring the surface heights at positions $1 \leqslant n$ $\leqslant L+1$ of the growth substrate. As it was mentioned above, we are interested in boundary conditions that suppress completely height fluctuations at $n=1$ and $L+1$ for all times, i.e., the interface is anchored at the boundaries. For simplicity, we study the case where $L$ is even and $h_{1}=h_{L+1}$, whereas deposition and evaporation rates $\epsilon, \epsilon^{\prime}$ are taken uniformly throughout the system except on site $L / 2+1$ where these probability values are respectively $\epsilon_{0}, \epsilon_{0}^{\prime}$. To prevent the divergence of interface fluctuations in the bulk, we impose a RSOS constraint, namely, $\left|h_{n+1}-h_{n}\right| \equiv 1, \forall$ $n, t$. Specifically, growth (evaporation) events $h_{n} \rightarrow h_{n}+2$, $\left[h_{n} \rightarrow h_{n}-2\right]$, with $n=2, \ldots, L$, occur only at local minima (maxima) of the evolving interface. These basic processes

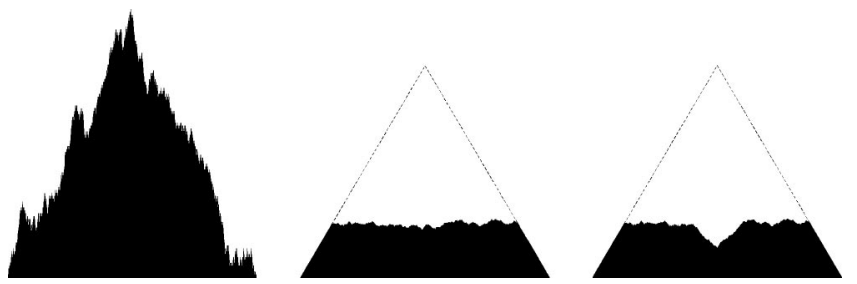

FIG. 2. Possible evolution scenarios. Typical snapshots for $L$ $=10^{3}$ using $\epsilon^{\prime} / \epsilon=1$ with $\epsilon_{0}^{\prime} / \epsilon_{0}=0.5$ after $t=10^{6}$ steps per height (left); $\epsilon^{\prime} / \epsilon=\epsilon_{0}^{\prime} / \epsilon_{0}=0.5$ at $t=500$ (center); and $\epsilon^{\prime} / \epsilon=0.5$ with $\epsilon_{0}^{\prime} / \epsilon_{0}=5$ at $t=500$ (right). For $\epsilon \neq \epsilon^{\prime}$, fluctuations are progressively reduced on their way to the pile configuration denoted by dotted lines with slopes \pm 1 .

and their transition rates are schematized in Fig. 1. In turn, the typical configurations resulting from these rules at large times are displayed by the snapshots of Fig. 2 .

It is often convenient to consider the interface slope rather than the height, so hereafter we will exploit the known mapping between RSOS interface dynamics and quantum spin- $\frac{1}{2}$ systems. This correspondence can be easily visualized in the scheme of Fig. 1. Associating the height difference $s_{n}$ $\equiv h_{n+1}-h_{n}$ to an eigenvalue of the $z$ component, say, of the Pauli operator $\vec{\sigma}_{n}$ for site $n$, all relevant quantities of the interface, such as its width and height-height correlations, can be casted in terms of $\frac{1}{2}$-spinors. By construction, it is clear that the interface heights (relative to $h_{1}$ ) are obtained as $h_{n}=\sum_{j=1}^{n-1} s_{j}$ for $n=2, \ldots, L+1$. Therefore, the anchoring condition $h_{1}=h_{L+1}$ imposes the vanishing of the total magnetization throughout the underlying spin kinetics.

As is well known, the probability distribution of such Markov processes is controlled by a master equation [12]

$$
\partial_{t} P(s, t)=\sum_{s^{\prime}}\left[R\left(s^{\prime} \rightarrow s\right) P\left(s^{\prime}, t\right)-R\left(s \rightarrow s^{\prime}\right) P(s, t)\right]
$$

whose transition probability rates $R\left(s \rightarrow s^{\prime}\right) \in\left\{\epsilon, \epsilon^{\prime}, \epsilon_{0}, \epsilon_{0}^{\prime}\right\}$ now denote the (biased) spin exchanges at which a generic configuration $|s\rangle \equiv\left|s_{1}, \ldots, s_{L}\right\rangle$ evolves to $\left|s^{\prime}\right\rangle$ through a single exchange of two consecutive spins. Starting from a given probability distribution $|P(0)\rangle=\Sigma_{s} P(s, 0)|s\rangle$, Eq. (4) can be conveniently thought of as a Schrödinger-like representation in which the ensemble averaged state vector $|P(t)\rangle$ (playing the role of wave function) can be evaluated at subsequent times from the action of an evolution operator (or Hamiltonian) on the initial state, namely, $|P(t)\rangle$ $=e^{-H t}|P(0)\rangle[13]$. The specific form of $H$ can be readily obtained by introducing spin- $\frac{1}{2}$ raising and lowering operators $\sigma^{+}, \sigma^{-}$, along with spin occupation fields $\hat{\mathbf{n}}=\sigma^{+} \sigma^{-}$. It is then straightforward to show that the stochastic dynamics of Eq.(4) is accounted for by the operator

$$
\begin{aligned}
H= & -\sum_{n=1}^{L-1}\left(\epsilon_{n} \sigma_{n}^{+} \sigma_{n+1}^{-}+\epsilon_{n}^{\prime} \sigma_{n+1}^{+} \sigma_{n}^{-}\right) \\
& +\sum_{n=1}^{L-1}\left[\epsilon_{n} \hat{\mathbf{n}}_{n+1}\left(1-\hat{\mathbf{n}}_{n}\right)+\epsilon_{n}^{\prime} \hat{\mathbf{n}}_{n}\left(1-\hat{\mathbf{n}}_{n+1}\right)\right],
\end{aligned}
$$


where $\epsilon_{n}, \epsilon_{n}^{\prime}$ are shorthands denoting, respectively,

$$
\epsilon_{n}, \epsilon_{n}^{\prime}=\left\{\begin{aligned}
\epsilon_{0}, \epsilon_{0}^{\prime} & \text { for } n=\frac{L}{2} \\
\epsilon, \epsilon^{\prime} & \text { otherwise }
\end{aligned}\right.
$$

while the anchoring condition $h_{1}=h_{L+1}$ confines the dynamics within the subspace $\Sigma_{n} \hat{\mathbf{n}}_{n} \equiv L / 2$. The biased hopping terms of Eq. (5) clearly describe the original growthdesorption events (see Fig. 1), while conservation of probability requires in turn the appearance of the remaining (diagonal) particle-vacancy correlators. We address the reader to Ref. [14] for a more detailed derivation in related systems.

\section{A. Detailed balance}

Before continuing with an alternative spin representation more suitable to study dynamical aspects at large times, we pause and consider the steady state (SS) of Eq. (5) along with its implications on the interface character.

Given two spin configurations $|s\rangle=\left|\ldots, s_{n},-s_{n}, \ldots\right\rangle$, $\left|s^{\prime}\right\rangle=\left|\ldots,-s_{n}, s_{n}, \ldots\right\rangle$, differing at most in the state of two neighboring $n, n+1$ locations, evidently detailed balance probabilities in Eq. (4) will hold provided that

$$
\begin{aligned}
& P(s) \epsilon_{n}^{\prime}=P\left(s^{\prime}\right) \epsilon_{n} \text { if }\left\langle s^{\prime}|H| s\right\rangle=-\epsilon_{n}^{\prime}, \\
& P(s) \epsilon_{n}=P\left(s^{\prime}\right) \epsilon_{n}^{\prime} \text { if }\left\langle s^{\prime}|H| s\right\rangle=-\epsilon_{n} .
\end{aligned}
$$

This can be readily satisfied defining a hard-core particle (up-spin) potential

$$
V(n)=\sum_{j=1}^{n} \ln \left(\frac{\epsilon_{j}}{\epsilon_{j}^{\prime}}\right)=n \ln \left(\frac{\epsilon}{\epsilon^{\prime}}\right)+\ln \left(\frac{\epsilon_{0} \epsilon^{\prime}}{\epsilon_{0}^{\prime} \epsilon}\right) \Theta(n-L / 2),
$$

through which the equilibrium distribution is simply obtained as

$$
P\left(s_{1}, \ldots, s_{L}\right) \propto \exp -\left[\frac{1}{2} \sum_{n} V(n)\left(1+s_{n}\right)\right] .
$$

When $\epsilon=\epsilon^{\prime}$, these probabilities further enable us to construct the partition function (normalization constant), height profiles (spin densities), as well as the spin correlation functions needed to derive the equilibrium interface width. For $\epsilon \neq \epsilon^{\prime}$ a rather involved recursive relation in the particle number can be obtained for all these quantities, but its analytic solution is not reachable by standard means [15]. However, this case does not yield a rough interface as statistical fluctuations become exponentially suppressed in time (see Sec. III B).

If $\epsilon=\epsilon^{\prime}$, the step function potential (8) permits to divide the system into two independent regions $[1, L / 2],[L / 2$ $+1, L]$ with $\left(\begin{array}{c}L / 2 \\ m\end{array}\right)$ configurations having $L / 2-m$ and $m$ particles, respectively $(0 \leqslant m \leqslant L / 2)$. Hence, the partition function normalizing the above $\mathrm{SS}$ distribution is given by

$$
Z=\sum_{m=0}^{L / 2}\left(\begin{array}{c}
\frac{L}{2} \\
m
\end{array}\right)^{2} r^{m}
$$

where $r=\epsilon_{0}^{\prime} / \epsilon_{0}$. Using analogous arguments, we can also obtain the reduced partition function $Z_{i}$ which arises from the occupation of a given site $i$, and evaluate the spin density $\left\langle\sigma_{i}^{z}\right\rangle=2 Z_{i} / Z-1$. This results in

$$
Z_{i}=\left\{\begin{array}{cc}
\sum_{m=0}^{L / 2-1}\left(\begin{array}{c}
\frac{L}{2}-1 \\
m
\end{array}\right)\left(\begin{array}{c}
\frac{L}{2} \\
m
\end{array}\right) r^{m} & \text { for } i \leqslant \frac{L}{2}, \\
\sum_{m=0}^{L / 2-1}\left(\begin{array}{c}
\frac{L}{2} \\
m+1
\end{array}\right)\left(\begin{array}{c}
\frac{L}{2}-1 \\
m
\end{array}\right) r^{m+1} \quad \text { otherwise }
\end{array}\right.
$$

which implies an equilibrium shock profile stemming entirely from the inhomogeneous potential (8) at finite particle densities. In particular, for $L \rightarrow \infty$ the analysis of Eqs. (10) and (11) yields the following discontinuity:

$$
\left\langle\sigma_{n}^{z}\right\rangle=[1-2 \Theta(n-L / 2)]\langle s\rangle, \quad\langle s\rangle=\frac{\sqrt{\epsilon_{0}}-\sqrt{\epsilon_{0}^{\prime}}}{\sqrt{\epsilon_{0}}+\sqrt{\epsilon_{0}^{\prime}}} .
$$

Thus, in the height representation, so long as $\epsilon_{0}^{\prime} / \epsilon_{0}<1$ $(>1)$ Eq. (12) entails the sideways growth of a hill (valley) whose sides at large times are tilted by an amount of $\pm\langle s\rangle$ relative to the substrate. Though anchoring conditions were used throughout, tilted profiles are also observable in nonequilibrium systems such as those considered in Ref. [4] using periodic boundary conditions.

To determine whether this morphology is actually rough, we focus attention on the equilibrium height fluctuations $\delta_{n}$ $\equiv\left\langle h_{n}^{2}\right\rangle-\left\langle h_{n}\right\rangle^{2}$. Consequently, first we evaluate the spin-spin correlations involved in $\left\langle h_{n}^{2}\right\rangle=(n-1)+2 \sum_{i<j \leqslant n-1}\left\langle\sigma_{i}^{z} \sigma_{j}^{z}\right\rangle$. Thus, once more we recur to the combinatorial reasoning and calculate the reduced partition functions $Z_{i, j}$ resulting from the occupation of two specific sites $i, j$. After some elementary steps we obtain

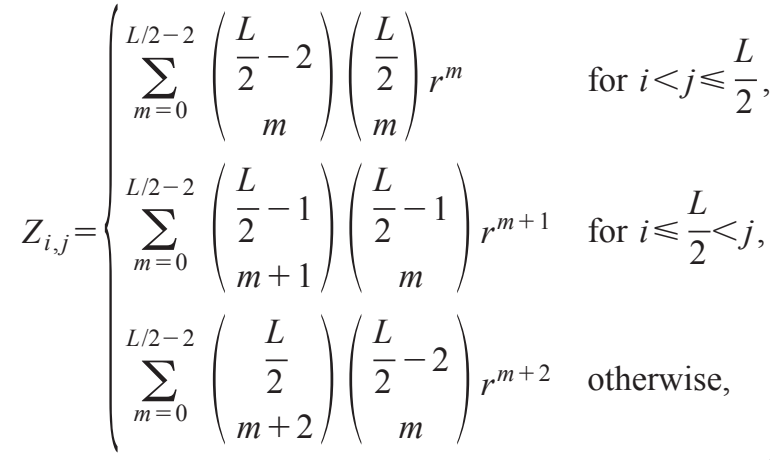

from which the required spin correlations are computed as $\left\langle\sigma_{i}^{z} \sigma_{j}^{z}\right\rangle=4 Z_{i, j} / Z-\left(\left\langle\sigma_{i}^{z}\right\rangle+\left\langle\sigma_{j}^{z}\right\rangle+1\right)$. In the large size limit the analysis of Eqs. (10), (11), and (13) ultimately yields a rough interface (see leftmost snapshot of Fig. 2), whose 


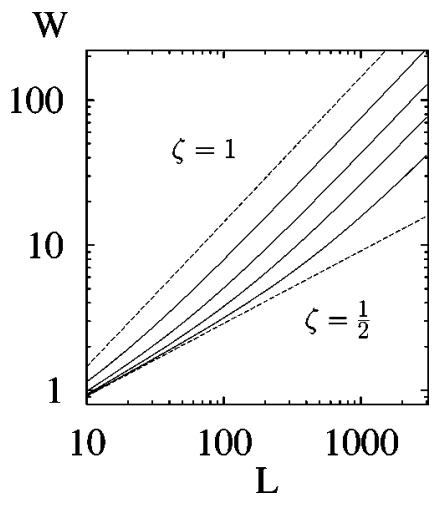

FIG. 3. Equilibrium interface width for $\epsilon / \epsilon^{\prime}=1$. Upper and lower dashed curves denote, respectively, the cases $\epsilon_{0}^{\prime} / \epsilon_{0}=0$ and 1, whereas solid lines going downwards stand for $\epsilon_{0}^{\prime} / \epsilon_{0}=0.1,0.3,0.5$, and 0.7 .

height fluctuations (canceled at the boundaries), result distributed as $\delta_{n} \propto(n-1)[1-(n-1) / L]$. This simple form is in contrast with that observed in nonequilibrium SS of anchored self-organized interfaces in which fluctuations in the upper part of the hill are substantially reduced [5].

The above correlations can also characterize the saturation width referred to in Sec. I. Specifically, in the spin representation it can be easily checked that $W^{2}$ may be rewritten as

$$
W^{2}(L)=\frac{L^{2}-1}{6 L}+\frac{2}{L^{2}} \sum_{i<j} i(L-j)\left\langle\sigma_{i}^{z} \sigma_{j}^{z}\right\rangle .
$$

In Fig. 3 we display the size dependence of $W$ for several values of $\epsilon_{0}^{\prime} / \epsilon_{0}$. It turns out that the roughness exponent $\zeta$ bears the discontinuous character of Eqs. (11) and (13). More precisely,

$$
\zeta=\left\{\begin{array}{lll}
1 & \text { if } & \epsilon_{0}^{\prime} \neq \epsilon_{0}, \\
1 / 2 & \text { if } & \epsilon_{0}^{\prime}=\epsilon_{0} .
\end{array}\right.
$$

Often, a value of $\zeta=1$ is special because it signals that the assumption of a well defined average orientation of the interface (parallel to the substrate plane) becomes inconsistent. Certainly, this is in line with the tilt obtained in Eq. (12). For $\epsilon_{0}^{\prime}=\epsilon_{0}$ the conventional roughening is recovered; here the tilt vanishes and the orientational fluctuations at large scales estimated, for example, as $W(L, t \rightarrow \infty) / L$, decrease with $L$. We shall revisit this point later on in Sec. III A.

\section{B. Self-adjoint representation}

As is known [12], detailed balance guarantees the existence of a representation in which the evolution operator (5) is self-adjoint. Although an exact solution of the (real) $H$ spectrum in the thermodynamic limit seems unlikely irrespective of its representation, at least a self-adjoint description can facilitate the numerical analysis of a finite size scaling approach. Specifically, one can readily find a similarity transformation to map Eq. (5) into a Hermitian matrix, and thereafter obtain the lower eigenmodes dominating the asymptotic kinetics via recursion-type algorithms, e.g., the Lanczos technique [16], appropriate to study fair system sizes.

To this aim and with the aid of the particle potential introduced in Eq. (8), we rotate the above operator around the $z$ spin direction using a pure imaginary site dependent argument $\varphi(n)$,

$$
\varphi(n)=\frac{i}{2} V(n) .
$$

This rotation is produced by the nonunitary similarity transformation $U=e^{-i S}$ with $S=\frac{1}{2} \Sigma_{n} \varphi(n) \sigma_{n}^{z}$, which in turn results in the direct product

$$
U=\otimes_{n} U_{n}, U_{n}=\left[\begin{array}{cc}
e^{1 / 4 V(n)} & 0 \\
0 & e^{-(1 / 4) V(n)}
\end{array}\right] .
$$

While the diagonal terms of Eq. (5) remain unaltered by $U$, it is straightforward to show that

$$
U_{n} \sigma_{n}^{ \pm} U_{n}^{-1}=e^{\mp i \varphi(n)} \sigma_{n}^{ \pm} .
$$

From this latter transformation, one can immediately verify that the rotated (self-adjoint) operator $\mathcal{H}=U H U^{-1}$ becomes an open $X X Z$ ferromagnet with a defect coupling under local magnetic fields, namely,

$$
\begin{aligned}
\mathcal{H}= & -\frac{1}{2} \sum_{n=1}^{L-1} J_{n}\left[\sigma_{n}^{x} \sigma_{n+1}^{x}+\sigma_{n}^{y} \sigma_{n+1}^{y}+\Delta_{n}\left(\sigma_{n}^{z} \sigma_{n+1}^{z}-1\right)\right] \\
& -h\left(\sigma_{1}^{z}-\sigma_{L}^{z}\right)-\left(h_{0}-h\right)\left(\sigma_{L / 2}^{z}-\sigma_{L / 2+1}^{z}\right)
\end{aligned}
$$

where

$$
\begin{gathered}
J_{n}=\sqrt{\epsilon_{n} \epsilon_{n}^{\prime}}, \\
\Delta_{n}=\left(\epsilon_{n}+\epsilon_{n}^{\prime}\right) / \sqrt{4 \epsilon_{n} \epsilon_{n}^{\prime}}, \\
h_{0}=\left(\epsilon_{0}-\epsilon_{0}^{\prime}\right) / 4, \\
h=\left(\epsilon-\epsilon^{\prime}\right) / 4,
\end{gathered}
$$

with $\epsilon_{n}, \epsilon_{n}^{\prime}$ taken as in Eq. (6). Thus, we are left with a diagonalization problem which, to some extent, is now controllable by standard recursive techniques (Sec. III).

For the sake of completeness it is worth pointing out that the similarity transformation (17) also enables us to obtain the SS distribution (9). In fact, exploiting that $H$ is a stochastic operator, we can express its left SS $\langle\widetilde{\psi}|$ as an equally weighted sum of all accessible configurations [12], i.e., $\langle\widetilde{\psi}$ $\mid \equiv\langle 0| \Sigma_{n_{1}}, \ldots, n_{L / 2} \sigma_{n_{1}}^{-} \cdots \sigma_{n_{L / 2}}^{-}$, where $\langle 0|$ denotes the ferromagnetic down-spin state. Hence, by construction $\left|\psi_{0}\right\rangle$ $=U^{-1}|\widetilde{\psi}\rangle$ is a (unnormalized) ground state of $\mathcal{H}$, and therefore the SS distribution in the initial $H$ representation is constructed as 


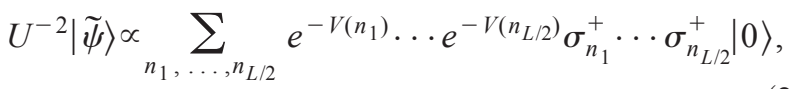

thus recovering the equilibrium distribution (9).

Returning to the dynamics, the average value of a diagonal quantity $\hat{\mathcal{W}}$ - such as the "width operator" involved in Eq. (14)—varies according to $\left\langle\widetilde{\psi}\left|\hat{\mathcal{W}} e^{-H t}\right| P(0)\right\rangle$ [14]. Since $\hat{\mathcal{W}}$ is invariant under $U$, it is a simple matter to check that in the self-adjoint representation $\langle\hat{\mathcal{W}}\rangle$ reads

$$
\langle\hat{\mathcal{W}}\rangle(L, t)=\left\langle\psi_{0}|\hat{\mathcal{W}}| \psi_{0}\right\rangle+\sum_{\lambda>0} e^{-\lambda t}\left\langle\psi_{0}|\hat{\mathcal{W}}| \psi_{\lambda}\right\rangle\left\langle\psi_{\lambda}|U| P(0)\right\rangle
$$

where $\left\{\left|\psi_{\lambda}\right\rangle\right\}$ is a complete orthonormal set of eigenstates of $\mathcal{H}$ (all with $\lambda \geqslant 0$ ). As expected, the role of initial conditions becomes irrelevant near the equilibrium regime. If the spectrum gap vanishes in the thermodynamic limit, the width approach to equilibrium will involve arbitrarily large times for sufficiently large systems. In those situations, finite size scaling analyses of the first excited levels $\lambda_{L}$ would then provide the dynamic $z$-exponent ruling over the late roughening stages referred to in Sec. I.

A distinctive feature arises when all components of the total angular momentum $\mathbf{S}=\frac{1}{2} \Sigma_{n} \vec{\sigma}_{n}$ are preserved by $\mathcal{H}$, namely, for $\epsilon=\epsilon^{\prime}$ and $\epsilon_{0}=\epsilon_{0}^{\prime}$. Since $\hat{\mathcal{W}}$ just involves operators of the form $\sigma_{i}^{z} \sigma_{j}^{z}$ [see Eq. (14)], then rather restrictive selection rules hold for its matrix elements in Eq. (22). Specifically, given that $\left\{\left|\psi_{\lambda}\right\rangle\right\}$ can be classified according to the total spin $S\left[\mathbf{S}^{2} \equiv S(S+1)\right]$, the nonvanishing contributions to Eq. (22) come only from states $\left|\psi_{\lambda}\right\rangle$ having $S=L / 2-1$ and $L / 2-2$ [17]. What should be emphasized here is that as soon as $[\mathcal{H}, \mathbf{S}] \neq 0$ the effective density of states, partly responsible for the temporal asymptotic behavior of Eq. (22) when $L \rightarrow \infty$, is drastically modified as these selection rules no longer apply. We will come back to this issue within the numerical context of Sec. III A.

\section{NUMERICAL RESULTS}

To explore the dynamical consequences of these arguments we have carried out Monte Carlo simulations as well as finite size scaling analyses of the RSOS model referred to above, for a variety of situations. First we focus attention on the subcase $\epsilon=\epsilon^{\prime}$ where the roles of $\epsilon_{0}$ and $\epsilon_{0}^{\prime}$ are clearly interchangeable, so we restrict the analysis to, say, $\epsilon_{0}^{\prime} / \epsilon_{0}$ $\leqslant 1$. In this situation the interface actually roughens and exhibits two different scaling regimes accompanying a large scale morphological transition. The discussion of different bulk probability rates is addressed at the second part of the section. It will turn out there that fluctuations decay very rapidly and prevent the roughening of anchored interfaces at large times. Instead, a faceting dynamics will emerge regardless of the inhomogeneity growth rates.

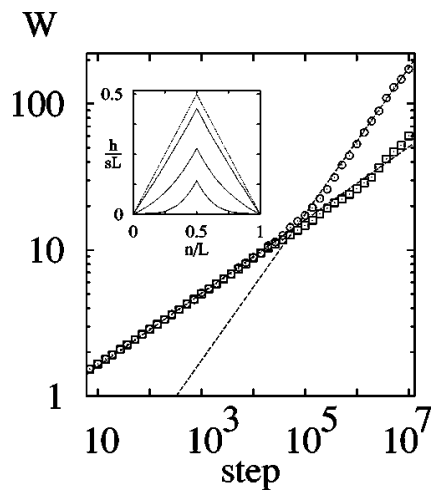

FIG. 4. Growth of interface width for $\epsilon / \epsilon^{\prime}=1$ using $\epsilon_{0}^{\prime} / \epsilon_{0}$ $=0.5$ (circles) and $\epsilon_{0}^{\prime} / \epsilon_{0}=0.8$ (squares showing an incipient asymptotic deviation) for $L=10^{4}$ averaged over 200 histories. The early and late slopes of dashed lines are, respectively, $\beta \simeq 1 / 4$ and $1 / 2$. The inset displays the height profile evolution for $\epsilon_{0}^{\prime} / \epsilon_{0}=0.5$ averaged over 2000 histories with $L=1000$, at $t=1.5 \times 10^{4}$, $6 \times 10^{4}, 2 \times 10^{5}$, and $10^{6}$ [the dotted line at the top following closely the tilt $s$ of Eq. (12)].

\section{A. $\boldsymbol{\epsilon}=\boldsymbol{\epsilon}^{\prime}$}

Starting from an initially flat configuration we studied the evolution of the interface width and monitored the height profiles at different growth stages. Figure 4 displays the width behavior obtained for $\epsilon_{0}^{\prime} / \epsilon_{0}=0.5$ and 0.8 . The rather slow crossover (particularly for 0.8 , where it is only incipient) deterred us from using larger substrates, though preliminary simulations averaged over few evolution samples indicated similar trends. The early growing stages support a power law growth $W \propto t^{\beta}$ extended over more than four decades with an exponent $\beta \simeq 1 / 4$. This typical diffusive behavior is accompanied initially by a height profile which is almost parallel to the substrate, except in the neighborhood of the growth inhomogeneity. As is shown in the inset of Fig. 4, on approaching the asymptopia however, the slopes of the hillsides steepen until they reach the equilibrium tilt alluded to in Eq. (12). This progressive orientation departure signals a large scale morphological transition which in turn is also reflected in the increase of the growth exponent. In the late dynamic stages, this can be well fitted by a value of $\beta$ $\simeq 1 / 2$ for nearly two decades.

An alternative determination of this rather peculiar value [18] can be implemented by resorting to the phenomenological scaling assumption referred to in Eq. (2) along with the findings and arguments of Sec. II. Notice that this provides two independent numerical procedures to estimate the dynamic $z$ exponent needed for the knowledge of $\beta$. On one hand, the former can be calculated by studying the finite size behavior of the first excited levels of $\mathcal{H}$ (in principle, just the lowest will do), so we diagonalized it exactly via a recursion type Lanczos algorithm [16] applied on the zero magnetization subspace. The huge dimensionality of this sector, growing as $\left(\begin{array}{c}L / 2 \\ L\end{array}\right)$, as well as the lack of translational symmetry of the evolution operator, limited our computations to chain lengths of up to 24 spins. Nevertheless, they proved to be sufficient for a fair estimation of the spectrum gap. We direct the reader to Fig. 5 which suggests a decrease $\propto L^{-z}$ for the 


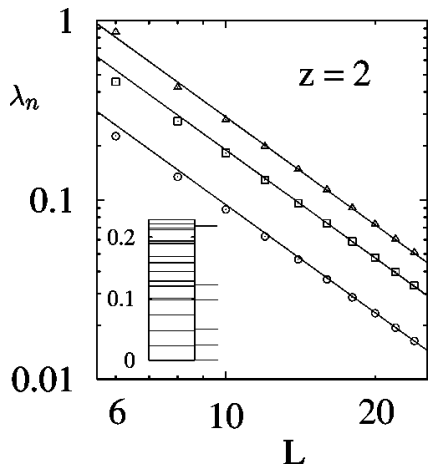

FIG. 5. Finite size behavior of the three lowest excited levels of the evolution operator (5) for $\epsilon / \epsilon^{\prime}=1$ and $\epsilon_{0}^{\prime} / \epsilon_{0}=0.5$. Solid lines have slopes $z=2$. The inset compares the lower spectrum of this case ( $L=20,10$ spin excitations, framed at the left), with the effective levels of the regular situation $\left(\epsilon_{0} / \epsilon_{0}^{\prime}=1, L=20,2\right.$ spin excitations).

gap and other excited levels consistent with a common value of $z=2$. On the other hand, an independent evaluation of this exponent can be attained by studying the scaling behavior of the interface width [Eq. (2)]. Figure 6 exhibits the results of our simulations for growth substrates of 2500 and 5000 heights on approaching their saturation regimes. Here, the data collapse was obtained upon setting $(z, \zeta) \simeq(2,1)$, which confirms not only the Lanczos estimation but also corroborates the roughness exponent quoted in Eq. (15). Thus, from Eq. (2) it follows that the fast roughening behavior already observed in Fig. 4 is now recovered by the ratio $\zeta / z$.

Since the Lanczos analysis continues to yield values of $z=2$ holding up to the homogeneous situation (as it should), a natural question one can pose is therefore the following: through which feature does the dynamics render a completely different roughening behavior at large times as soon as $\epsilon_{0} \neq \epsilon_{0}^{\prime}$ ? In an attempt to provide a plausible explanation for the appearance of this abrupt change [see also Eq. (15)], we resort to the observations given by the end of Sec. II B.

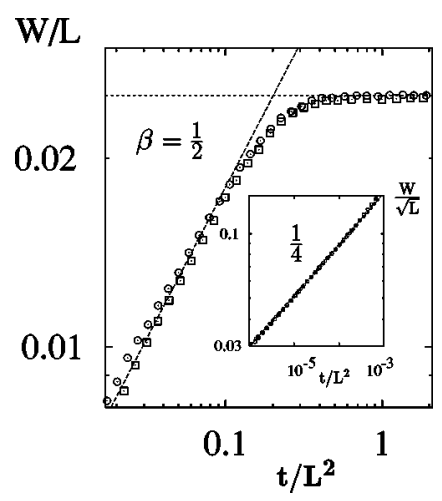

FIG. 6. Asymptotic finite size scaling regime of the interface width averaged over 200 histories using $\epsilon / \epsilon^{\prime}=1, \epsilon_{0}^{\prime} / \epsilon_{0}=0.5$, for $L=5000$ (squares), and $L=2500$ (circles). The saturation values (horizontal line) coincide with those of Eqs. (13) and (14). The dashed line is fitted with the slope calculated from the data of Figs. 3 and 5 (i.e., $\beta=\zeta / z$ ). The inset exhibits an early scaling regime which behaves diffusively $(\beta=1 / 4)$, like the initial data of Fig. 4 .
The inset of Fig. 5 displays the lower part of the $\mathcal{H}$ spectrum for both inhomogeneous and homogeneous situations. As stated above, the latter involves at most $L(L-1) / 2$ contributing levels with $S=L / 2-1, L / 2-2$, while in the former case the sum of Eq. (22) becomes much denser as $[\mathcal{H}, \mathbf{S}]$ $\neq 0$ and an exponential number of new states arises. Of course, the new matrix elements might eventually change from zero in a continuous manner, but the density of states (a measure of which is given by the inverse of the level spacing) varies abruptly. Moreover, some of the low lying excitations controlling the asymptotic regime $(t \rightarrow \infty$ holding $\left.t / L^{z} \ll 1\right)$ suggest a rather narrowly peaked structure which is entirely absent for $\epsilon_{0}=\epsilon_{0}^{\prime}$.

Also, it is interesting to examine whether the large scale morphology transition embodied in the slow temporal crossover of $W$ affects the usual scaling hypothesis of Eqs. (2) and (3). Thus, we turn to the early dynamic scaling of $W$ shown by the inset of Fig. 6. In contrast to the faster growth observed at late stages, here the data collapse arises by setting standard diffusive exponents $(z, \zeta) \simeq(2,1 / 2)$, which in turn yield a scaling function $\propto\left(t / L^{2}\right)^{1 / 4}$ in agreement with the early $\beta$ exponent measured in Fig. 4 . Hence, combining the late and early scaling regimes it follows that

$$
W(L, t, \tau)=L^{\zeta} f_{\tau}\left(t / L^{2}\right),
$$

where $\tau$ is a crossover time which depends solely on $\epsilon_{0}^{\prime} / \epsilon_{0}$ (eventually diverging in the limit $\epsilon_{0}^{\prime} \rightarrow \epsilon_{0}$ ) and $f_{\tau}(c)$ is a universal function defined over three different scales as

$$
f_{\tau}(c) \sim\left\{\begin{array}{lll}
c^{1 / 4} & \text { for } \quad c \ll \tau / L^{2} & (\zeta=1 / 2) \\
c^{1 / 2} & \text { for } \quad \tau / L^{2} \ll c \ll 1 & (\zeta=1) \\
\text { const } & \text { for } c \gg 1 . &
\end{array}\right.
$$

In what follows we finally address the $\epsilon \neq \epsilon^{\prime}$ situation.

$$
\text { B. } \epsilon \neq \epsilon^{\prime}
$$

A quick glance at the evolution of the interface width displayed in Fig. 7 might render the (wrong) impression that the general case $\epsilon \neq \epsilon^{\prime}, \epsilon_{0} \neq \epsilon_{0}^{\prime}$ bears similar characteristics. However this time the growth exponent exhibits an asymptotic value of $\beta \simeq 3 / 2$, which is not understandable in terms of conventional kinetic roughening theories [18]. In fact, as we shall see below, the interface does not roughen.

Let us first provide a simple explanation for this large value of $\beta$ by means of the following heuristic considerations. For clarity of argument, assume vanishing desorption rates and $\epsilon=\epsilon_{0}$. Starting from a flat configuration, say with $h_{2 k}=1, h_{2 k-1}=0$, a deterministic dynamics arises as $(L$ $-2) / 2$ deposition attempts occur on the initial $(L-2) / 2$ interface minima. Next, we are left with $h_{2 k}=1, h_{2 k-1}=2, h_{1}$ $=h_{L+1} \equiv 0$, and $(L-4) / 2$ contiguous minima over which new $(L-4) / 2$ depositions will be once again deterministic. By iterating this argumentation $t$ times, this dynamics leads to a configuration resembling a truncated pyramid (see central snapshot of Fig. 2, though for a nondeterministic situation, i.e., $\left.\epsilon^{\prime} \neq 0\right)$. Specifically, there will be $(L-2 t) / 2$ contiguous minima in between $h_{t+2}=t+1$ and $h_{L-t-1}=h_{t+2}$. 


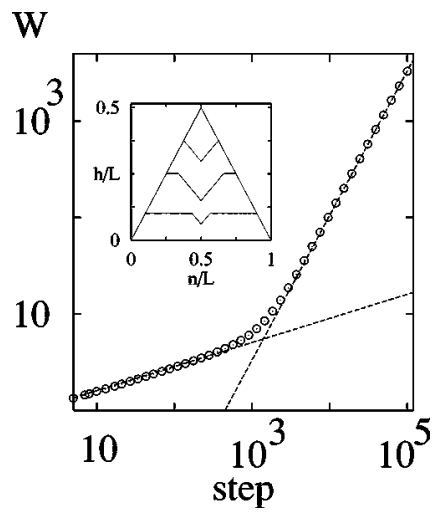

FIG. 7. Evolution of the interface width for $\epsilon^{\prime} / \epsilon=0.5, \epsilon_{0}^{\prime} / \epsilon_{0}$ $=5$, and $L=10^{6}$. The initial and final slopes of dashed lines are $\beta=1 / 4$ and $3 / 2$, respectively. From top to bottom the inset exhibits the profile of $10^{4}$ heights at $t=5 \times 10^{4}, 2 \times 10^{4}, 10^{4}$, and $4 \times 10^{3}$. Both width and height fluctuations become negligible at large times. At the width level, results of different $\epsilon_{0}^{\prime} / \epsilon_{0}$ values closely follow each other in all evolution stages. In turn, for $\epsilon_{0}^{\prime} \leqslant \epsilon_{0}$ the early profile has no tilt around the inhomogeneity.

Using the common definition of Eq. (1), it is then easy to verify that the "width" of such configuration is simply

$$
W(L, t)=\sqrt{\frac{2 t^{3}}{3 L}}[1+O(t / L)], \text { i.e., } \beta=3 / 2 .
$$

The above argument describes rather a faceting process (terminating at $t \sim L / 2$ ), which strictly applies for $\epsilon^{\prime}=0$. Certainly, as soon as $\epsilon^{\prime}>0$ the dynamics is no longer deterministic, no matter how small $\epsilon^{\prime}$ is. However, for $\epsilon^{\prime} \ll 1$ the early desorption attempts become gradually unsuccessful as the active region of the interface, i.e., the number of available minima decreases inasmuch as the sideways region is increasingly jammed (Fig. 2). Thus, at large times a process similar to a faceting dynamics might be expected, at least for small bulk desorption rates. In fact, for $\epsilon>\epsilon^{\prime}$ and $\epsilon_{0} \geqslant \epsilon_{0}^{\prime}$ our numerical simulations confirm these considerations. No differences were observed between sampling histories at large times, so fluctuations become asymptotically negligible. For $\epsilon_{0}<\epsilon_{0}^{\prime}$ the situation is similar though another faceting process shows up around the inhomogeneity, as displayed by the height profiles in the inset of Fig. 7 (see also rightmost snapshot of Fig. 2). Of course, for $\epsilon<\epsilon^{\prime}$ the roles of $\epsilon_{0}$ and $\epsilon_{0}^{\prime}$ are interchanged. Ultimately, the whole process approaches a nonfluctuating pile of slope \pm 1 , so long as $\epsilon^{\prime} \neq \epsilon$.

To provide an alternative understanding of this fast fluctuation decay for generic rate values, we recur once more to the analysis of the spectrum of the evolution operator (19). The gap and levels obtained for the sizes within our reach are shown in Fig. 8, but in contrast to the $\epsilon=\epsilon^{\prime}$ situation, here the finite size trend of these quantities needs further analysis. To this end, we studied the $\mathcal{H}$ spectrum within the subspace $S^{z}=L / 2-1$ (single spin excitation), which corresponds to the much simpler anchoring case $h_{L+1}-h_{1}=L-2$. For this sector, it is straightforward to check that Eq. (19) reduces to the tridiagonal matrix

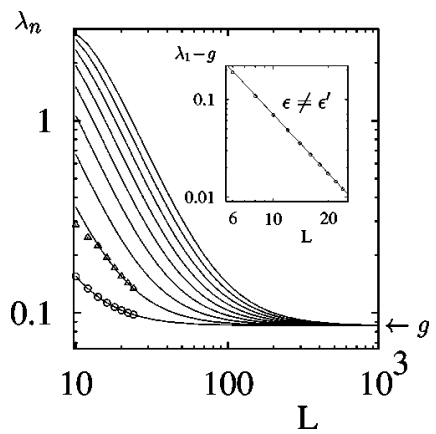

FIG. 8. Finite size behavior of lower excited levels in the single spin approximation $\left(S^{z}=L / 2-1\right)$ of operator (26) for $\epsilon^{\prime} / \epsilon=\epsilon_{0}^{\prime} / \epsilon_{0}$ $=1 / 2$ (solid lines). Circles and triangles stand, respectively, for levels $\lambda_{1}$ and $\lambda_{2}$ of Hamiltonian (5) with $S^{z}=0$. Above $\lambda_{2}$, further collective excitations (not shown) appear between successive solid lines. The inset suggests a power law convergence of $\lambda_{1}(L)$ (circles, $S^{z}=0$ ) towards the gap $g$ obtained in the main panel.

$\mathcal{H}=\left[\begin{array}{cccccccc}\epsilon^{\prime} & \gamma & 0 & \cdots & & & \cdots & 0 \\ \gamma & \epsilon+\epsilon^{\prime} & \gamma & \ddots & & & & \vdots \\ 0 & \ddots & \ddots & \ddots & & & & \\ \vdots & \ddots & \gamma & \epsilon+\epsilon_{0}^{\prime} & \gamma & 0 & & \\ & & 0 & \gamma_{0} & \epsilon^{\prime}+\epsilon_{0} & \gamma & \ddots & \vdots \\ & & & & \ddots & \ddots & \ddots & 0 \\ \vdots & & & & \ddots & \gamma & \epsilon+\epsilon^{\prime} & \gamma \\ 0 & \cdots & & & \ldots & 0 & \gamma & \epsilon\end{array}\right]$,

where

$$
\gamma=-\sqrt{\epsilon \epsilon^{\prime}}, \quad \gamma_{0}=-\sqrt{\epsilon_{0} \epsilon_{0}^{\prime}}
$$

Evidently, this just constitutes the simplest approximation to the many body problem of $S^{z}=0$. Nevertheless, the comparisons of Fig. 8 indicate that the eigenvalues of Eq. (26) yet provide an excellent estimation of the actual gap and other excited levels obtained through the Lanczos scheme. (It is worth pointing out in passing that an excellent fit of these quantities was also found for $\epsilon=\epsilon^{\prime}$ ). Using the spectrum gap of the single spin approximation, the inset of Fig. 8 strongly suggests that the same gap will persist for $\lambda_{1}$ in the thermodynamic limit of Eq. (19). Similar gapful results were obtained for other values of $\epsilon_{0}^{\prime} / \epsilon_{0}$. Thus, fluctuations would be suppressed at large times, which is in line with the almost invariant values of $W$ observed over many sample histories.

Also, at the gap level the density of states diverges as $L$ in the simplified version of the problem. For $S^{z}=0$ however, one might conjecture a much stronger divergence, probably growing like $\sim e^{L}$, as the number of levels between two single excitations tends to increase exponentially with the system size (at least for the small lengths at hand). This would leave us with a saturation time $\propto L$ in Eq. (22), which on the other hand would be in agreement with the termination time of the faceting process idealized above. In fact, the numerical simulations displayed in Fig. 9 lend further sup- 


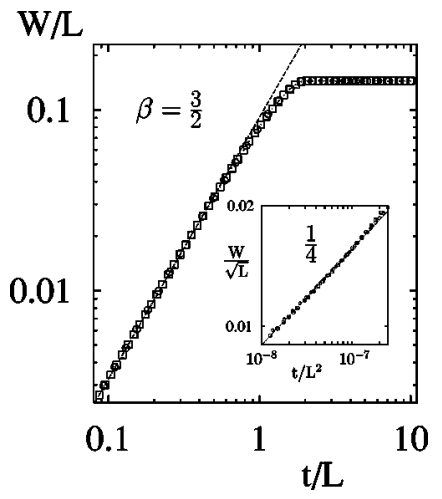

FIG. 9. Late scaling regime of $W$ for $\epsilon^{\prime} / \epsilon=\epsilon_{0} / \epsilon_{0}^{\prime}=0.5$ using $L=10^{4}$ (circles) and $L=2 \times 10^{4}$ (squares). The dashed line slope and scaling form are both consistent with the faceting process conjectured in Eq. (25), whereas differences between sample histories become gradually negligible. In contrast, the inset results, averaged over 200 histories, indicate an early scaling regime which is typically diffusive $(\zeta=1 / 2, z=2, \beta=\zeta / z)$.

port to these speculations. Clearly, these results exhibit both a saturation time $\propto L$, as well as an asymptotic scaling regime consistent with Eq. (25), i.e., $W / L \propto(t / L)^{3 / 2}$, during which both height and width fluctuations are absent.

In contrast, at early stages the interface displays typical roughening features. Specifically, the inset of Fig. 9 exhibits a diffusive scaling regime $W / \sqrt{L}=f\left(t / L^{2}\right)$, in turn corroborated by the growth exponent $\beta \simeq 1 / 4$ obtained in larger systems (Fig. 7). This strong departure from the faceting description occurs on temporal scales smaller than a crossover time which turns out to decrease when $\epsilon^{\prime} / \epsilon \rightarrow 0$ (irrespective of $\epsilon_{0}^{\prime} / \epsilon_{0}$ ), but eventually diverging in the limit $\epsilon^{\prime} \rightarrow \epsilon, \epsilon_{0}^{\prime}$ $\rightarrow \epsilon_{0}$.

\section{CONCLUSIONS}

We have analyzed the characteristics of both early and asymptotic dynamics of one-dimensional anchored interfaces under a growth inhomogeneity. There are two sets of results related, respectively, to equal or different growth-evaporation rates in the bulk.

For $\epsilon=\epsilon^{\prime}$, even the slightest departure from the homogeneous $\epsilon_{0}=\epsilon_{0}^{\prime}$ situation is able to produce finite interface tilts as well as huge temporal crossovers. The problems posed by the latter have been bypassed studying separately the roughness $\zeta$ and dynamic $z$ exponents, the evaluation of which was significantly simplified by the appearance of equilibrium SS. In analyzing finite size scaling trends of the spectrum gap via the Lanczos method, we found no changes with respect to the homogeneous situation, i.e., $z \equiv 2$. Consequently, it was argued that the breaking of the full rotational invariance of the evolution operator is ultimately responsible for the emergence of a much heavier density of states accounting for both the discontinuity of the roughness exponent $\zeta$ (or alternatively, for a different asymptotic growth exponent $\beta$ ) as well as for the rise of a new scaling regime at large times. These expectations were confronted independently with standard numerical simulations monitoring the evolution of the interface profile and width. At early stages, the latter exhibits a diffusive scaling regime having basically a nontilted profile (except in the inhomogeneity neighborhood), but progressively approaching a final regime consistent with our scaling exponents $\zeta=1, z=2$. However for $\epsilon_{0} \rightarrow \epsilon_{0}^{\prime}$, in practice this new regime might occur at a time so large as to render it numerically unobservable.

For $\epsilon \neq \epsilon^{\prime}$ the situation is entirely different. Here, the spectrum gap does not vanish in the thermodynamic limit regardless of the inhomogeneity rates, and fluctuations between evolution histories at large times become negligible. This confirms a heuristic description (in turn, tested independently by simulations), suggesting that the asymptotic dynamics becomes almost deterministic. We may also think of a synchronous discrete time process in which a randomly chosen finite fraction, or possibly all of the growth sites, are simultaneously updated in a single time step. One characteristic feature of such synchronous models is the occurrence of faceting transitions at large times [1], which also turned out to be the case here. In contrast, at early stages the interface actually roughens following a typical diffusive pattern accompanied by a standard scaling regime.

The analysis of nonequilibrium asymptotic situations, even for $d=1$, might become rather involved. In this sense, it will be interesting to elucidate whether a direct evaluation of $\zeta$ could be achieved using the matrix approach to the asymmetric exclusion process [19] with both injection and ejection of particles at the boundaries, including one or more hopping defects (that is, unanchored boundaries and growth inhomogeneities in the height representation).

Higher dimensional extensions of this study would be clearly desirable and more realistic. However, the analysis of the corresponding quantum spin analogy should involve a projector operator to discard all those spin configurations having magnetic loops, i.e., $\Sigma_{\mathbf{r}} \sigma_{\mathbf{r}}^{z} \neq 0$, with $\mathbf{r}$ on a given closed path. Otherwise, the mapping would no longer represent an interface. The issue as to whether or not such ideas are actually practical in $d>1$ remains quite open.

\section{ACKNOWLEDGMENT}

The author acknowledges support of CONICET, Argentina.
[1] For comprehensive reviews and literature list, consult J. Krug, Adv. Phys. 46, 139 (1997); T. Halpin-Healy and Y.-C. Zhang, Phys. Rep. 254, 215 (1995); P. Meakin, ibid. 235, 189 (1993); J. Krug and H. Spohn, in Solids far from Equilibrium: Growth,
Morphology and Defects, edited by C. Godréche (Cambridge University Press, Cambridge, 1992).

[2] However, the universality issue has been steadily brought into question, particularly in higher dimensions. For instance, see 
T.J. Newman and M.R. Swift, Phys. Rev. Lett. 79, 2261 (1997).

[3] M.C. Bartelt and J.W. Evans, J. Phys. A 26, 2743 (1993); B.D. Lubachevsky, V. Privman, and S.C. Roy, Phys. Rev. E 47, 48 (1993); H.C. Kang and J.W. Evans, Surf. Sci. 271, 321 (1992).

[4] D.E. Wolf and L.-H. Tang, Phys. Rev. Lett. 65, 1591 (1990).

[5] J. Krug, J.E. S Socolar, and G. Grinstein, Phys. Rev. A 46, R4479 (1992); J. Krug and J.E.S. Socolar, Phys. Rev. Lett. 68, 722 (1992); J. Krug and L.-H. Tang, Phys. Rev. E 50, 104 (1994).

[6] P. Meakin, P. Ramanlal, L.M. Sander, and R.C. Ball, Phys. Rev. A 34, 5091 (1986).

[7] M. Plischke, Z. Rácz, and D. Liu, Phys. Rev. B 35, 3485 (1987).

[8] G. Forgacs, R. Lipowsky, and Th. M. Nieuwenhuizen, in Phase Transitions and Critical Phenomena, edited by $\mathrm{C}$. Domb and J.L. Lebowitz (Academic, London, 1991), Vol. 14.

[9] L.-H. Tang and I.F. Lyuksyutov, Phys. Rev. Lett. 71, 2745 (1993).

[10] M. Kardar, G. Parisi, and Y.-C. Zhang, Phys. Rev. Lett. 56, 889 (1986).

[11] F. Family and T. Vicsek, J. Phys. A 18, L75 (1985). However, for an account on anomalous scaling, see J.M. López, Phys. Rev. Lett. 83, 4594 (1999).

[12] N.G. van Kampen, Stochastic Processes in Physics and Chem- istry, 2nd ed. (North-Holland, Amsterdam, 1992).

[13] This strategy in related systems can be traced back over more than two decades. For a review see D.C. Mattis and M.L. Glasser, Rev. Mod. Phys. 70, 979 (1998).

[14] G.M. Schütz, in Phase Transitions and Critical Phenomena, edited by C. Domb and J.L. Lebowitz (Academic, London, 2000); M.D. Grynberg and R.B. Stinchcombe, Phys. Rev. E 61, 324 (2000).

[15] H.S. Wilf, Generatingfunctionology (Academic, New York, 1994).

[16] For an account of this time honored technique see, for example, G.H. Golub and C.F. van Loan, Matrix Computations, 3rd ed. (Johns Hopkins University Press, Baltimore, 1996).

[17] S. Alexander and T. Holstein, Phys. Rev. B 18, 301 (1978); A.R. Edmonds, Angular Momentum in Quantum Mechanics, 2nd ed. (Princeton University Press, Princeton, NJ, 1960).

[18] In the continuum description of growth equations, $\beta=1 / 2$ has been conjectured as the upper random deposition limit of stochastic roughening. See Ref. [1].

[19] For a review, consult B. Derrida and M. Evans in Nonequilibrium Statistical Mechanics in One Dimension, edited by V. Privman (Cambridge University Press, Cambridge, 1996); see also R.B. Stinchcombe and G.M. Schütz, Phys. Rev. Lett. 75, 140 (1995) 